\title{
Spectral analysis of US Space Catalog ephemerides for LAGEOS-1
}

\author{
Isaias A. Araya* and Davide Amato ${ }^{\dagger}$ \\ Imperial College London, London SW7 2AZ, United Kingdom
}

\begin{abstract}
The US Space Catalog is the primary source of public ephemeris data on Resident Space Objects. Catalog ephemerides are published as Two-Line Elements (TLEs) which were originally defined as singly-averaged elements according to the Simplified General Perturbations 4 (SGP4) theory. Recently, TLEs have been derived through numerical fits to underlying special perturbations solutions. We conjecture that the numerical fit results in short-periodic terms being embedded in TLEs, and analyse the amplitude spectrum of piecewise continuous osculating solutions derived from TLE sequences for the LAGEOS-1 satellite. The orbital elements spectra contain short-periodic terms at frequencies larger than those reconstructed by the SGP4 theory, which supports the conjecture. For LAGEOS-1, short-periodic terms in addition to those already considered by SGP4 should not be added to TLEs in an effort to improve their accuracy, as they are likely to have been already partially embedded in the TLEs.
\end{abstract}

\section{Introduction}

$\mathrm{T}$ HE accurate and complete knowledge of ephemerides of the increasing population of Resident Space Objects (RSOs) is the basic requirement underpinning future developments in Space Situational Awareness (SSA). To this date, the US Space Catalog is still the most complete public source of RSO ephemerides, and is an important source of data both for satellite operators and commercial SSA service providers. The characterization of the accuracy of the US Space Catalog is therefore still a crucial matter. Ephemerides in the US Space Catalog are distributed publicly as singly-averaged elements compatible with the SGP4 (Simplified General Perturbations 4) general perturbations theory. The particular heritage format in which these element sets have these been distributed leads to their designation as Two-Line Elements (TLEs). Therefore, in the rest of this paper we will take "TLE" as a shorthand for SGP4 mean elements, although as of now (2021) the preferred format for the distribution of these elements is JSON.

The SGP4 theory ultimately traces its lineage to the solutions of the main satellite problem by Brouwer [1] and Kozai [2]. The 1959 Brouwer solution was extended by Brouwer and Hori [3] to include dissipative effects from drag from an exponential atmosphere. Another important contribution within the body of work descended from Brouwer's theory is that of Lyddane [4], who modified Brouwer's theory to remove singularities for small eccentricities and inclinations. Subsequently, Lane [5] and Lane and Cranford [6] replaced the exponential density law in the theory by Brouwer and Hori with a power law, which removed the need for the series expansion of the density function, and further removed limitations for small eccentricities and inclinations. The works by Lane and Cranford were used to formulate the AFGP4 general perturbation theory, which included effects from the first five zonal harmonics of the geopotential. SGP4 is obtained from the AFGP4 theory after retaining only certain periodic terms due to drag and by simplifying geopotential terms by making the assumption of small eccentricity [7]; in fact, only terms that do not contain the eccentricity as a factor and are of first order in $J_{2}$ are retained in SGP4 [8]. At the end of the 1970s, SGP4 was extended to model more accurately "deep-space" orbits with periods larger than $225 \mathrm{~min}$; this was achieved through the analytical modelling of long-periodic lunisolar effects and a semi-analytical solution for orbits within $12 \mathrm{~h}$ and $24 \mathrm{~h}$ resonances. The complete equations for SGP4 were disseminated by Hoots and Roehrich [9]. In 2006, Vallado et al. [10] released a revised SGP4 code that clarified a few differences in implementations that arose between different users; this is also the SGP4 code that we use in the present work. Recently, a new general perturbations theory designated as SGP4-XP (SGP4 - extended perturbations) has been developed to improve the performance of SGP4 across all orbital regimes [11].

An often overlooked aspect of SGP4 is its treatment of short-periodic terms, which are exclusively due to the Earth's oblateness. As with the long-periodic terms, only first-order terms in $J_{2}$ not containing the eccentricity are retained

\footnotetext{
*Undergraduate student, Department of Aeronautics. Currently: Quantitative Analyst, Bank of America, London, United Kingdom.

${ }^{\dagger}$ Lecturer, Department of Aeronautics. AIAA Member. Email: d.amato@imperial.ac.uk

*US Space Catalog ephemerides are distributed through https://wWw.space-track.org and https://celestrak.com/ As of November 2021, the latter website also provides ancillary services such as TLE validation, orbit visualization, and conjunction analysis.
} 


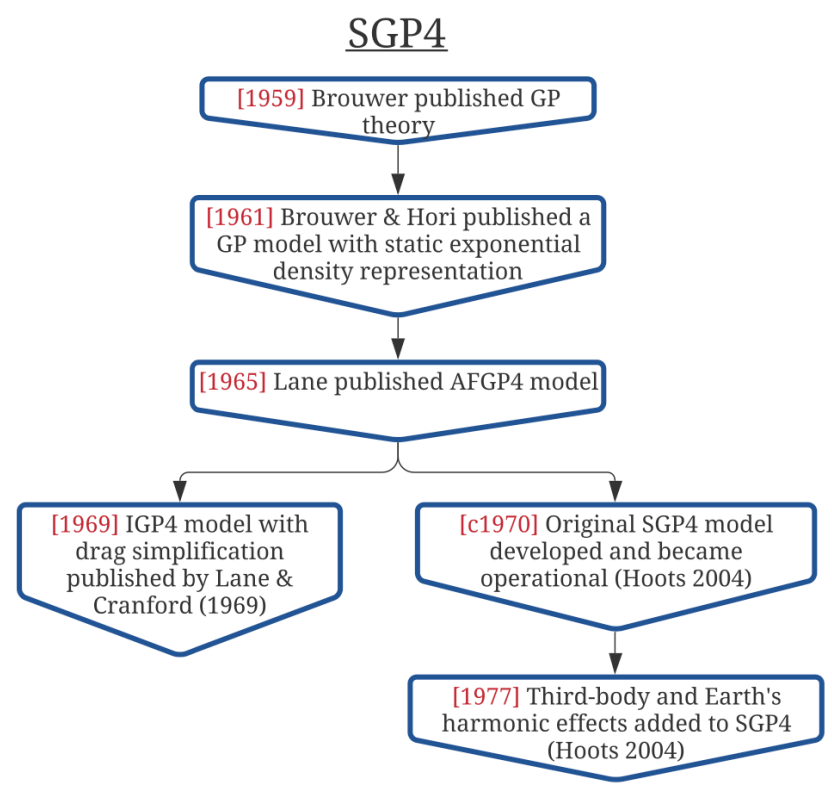

Fig. 1 Historical evolution of SGP4 with main references.

in SGP4. Unlike the original theory by Brouwer, short-periodic terms are directly added to the position and velocity vectors directly, likely to avoid singularities for small eccentricity [12, 13]. This is done by updating the inclination and right ascension of ascending node, which lead to the osculating angular momentum unit vector. Short-periodic terms are also added to the magnitudes of radius, radial velocity, angular velocity, and to the eccentric argument of latitude $u=E+\varpi \dagger^{\dagger}$ Finally, the osculating position and velocity vectors are computed in an Earth-centred frame using classical two-body relations. An important consequence for the purposes of the present work is that the short-periodic terms retained in SGP4 have frequencies that do not exceed $2 \dot{u}=2(\dot{\varpi}+\dot{v})$, which is approximately equal to $2 n$ for quasi-circular orbits.

It is well known that the US Space Force has been maintaining the catalog through special perturbations (SP) since at least May 2005 [14], following the successful implementation of SP methods on distributed computing platforms [15]. This high-accuracy catalog is also used to issue conjunction warnings to spacecraft operators [16]. Nevertheless, spacecraft ephemerides are still disseminated to the public as mean elements. At the moment, it is likely that TLEs are obtained through a numerical fit to the SP solutions, in which each TLE is adjusted in order to minimise the error between the respective osculating state (after adding SGP4 short-period terms) and the SP solution stored in the high-accuracy catalog. Effectively, SP vectors are treated as observations in a differential correction process [17--20]. The advantage of this method is that the TLE accuracy can be increased without any need of changing SGP4-based code on the end-user side. The consequence of this process is that (discounting noise) the corrections applied to TLEs in order to fit them to SP vectors correspond by definition to the short-periodic terms neglected by the SGP4 theory. Therefore, although historically related to the SGP4 theory, we conjecture that modern TLEs should be regarded as a form of osculating elements, as they contain short-periodic terms that have been reconstructed numerically. The goal of this paper is to take a first step towards experimentally proving this conjecture by analysing the amplitude spectrum of TLE series. To simplify the analysis, we focus on the trajectory of LAGEOS-1, which travels on a medium Earth orbit that is not affected by either drag or solar radiation pressure, and for which third-body perturbations are relatively small.

We highlight that spectral analysis methods have been applied in Solar System dynamics to prove that the dynamics of the major planets are chaotic [21, 22]. Spectral processing techniques have also been devised by Knežević and Milani [23] to compute asteroid synthetic proper elements, which have allowed generating catalogs of asteroid families deriving from individual parent bodies [24]. In the realm of Earth satellite dynamics, Ely [25] and Dell'Elce and others [26] devised mean-osculating transformations based on the discrete Fourier transform (DFT). Rosengren and others also proposed using DFTs to derive synthetic proper elements for RSOs [27, 28]. These could be eventually employed to associate breakup debris to their parent body [29], and for manoeuvre detection.

\footnotetext{
${ }^{\dagger}$ We use the non-standard notation $\varpi$ for the argument of perigee to avoid confusion with the frequency variable $\omega$.
} 


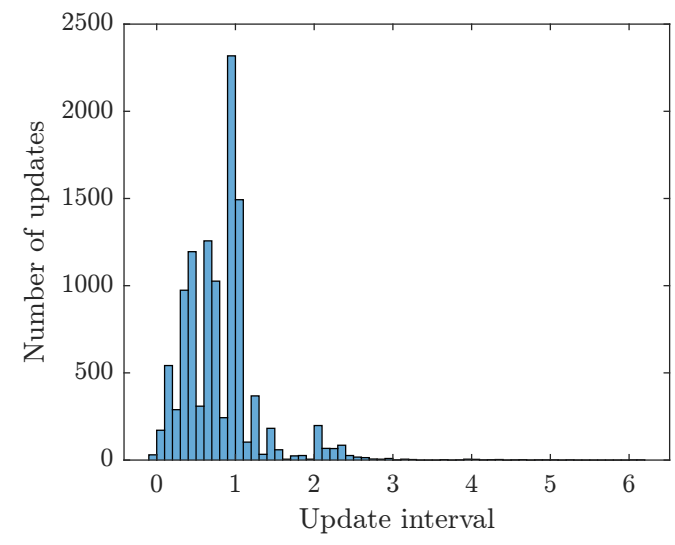

Fig. 2 Histogram of update frequencies for LAGEOS-1 TLEs between $24^{\text {th }}$ February 1996 and $24^{\text {th }}$ February 2021.

\section{Generation of osculating solutions from Two-Line Elements series}

The classical discrete Fourier transform requires samples to be uniformly spaced; however, TLE sequences are updated at irregular intervals. As shown in the histogram in fig. 2, typical TLE update intervals range from hours up to a few days. Even if TLEs were spaced at uniform intervals of a few hours, such a low updating frequency would prevent the accurate reconstruction of short-periodic terms in the amplitude spectrum as a consequence of the Nyquist-Shannon sampling theorem [30]. The theorem states that if the Nyquist frequency $\omega_{\mathrm{c}}$ is the maximum frequency of the harmonics contained in a band-limited time series, the sampling interval must satisfy

$$
\Delta t \leq \frac{1}{2 \omega_{\mathrm{c}}}
$$

for the discrete Fourier transform to exactly represent the original time series. If the signal is not band-limited, or if the condition above is not satisfied, then the energy content of frequencies higher than the sampling frequency $1 / \Delta t$ will shift to lower frequencies. The phenomenon is called aliasing, and results in spurious peaks being introduced in the amplitude spectrum. The maximum frequency of the short-periodic terms reconstructed by SGP4 is approximately $2 n$; therefore, the requirement on the sampling interval becomes $\Delta t<n / 4 \approx 1 \mathrm{~h}$, which is much shorter than the typical TLE update interval ${ }^{\circ}$

To circumvent the Nyquist limit and generate a uniformly spaced time series of orbital elements on which to perform a spectral analysis, we synthesise a piecewise continuous osculating solution from the SGP4 solution in mean elements. Such a "synthetic" solution effectively underlies pseudo-orbit determination techniques that have been proposed to enhance TLE accuracy [31, 32]. The process is sketched in fig. 3, and takes place according to the following steps:

1) We retrieve a time series of TLEs $\overline{\boldsymbol{x}}\left(\tau_{i}\right), i=0,1, \ldots$, The time series is not uniformly spaced.

2) We generate a uniformly spaced discretization of the time interval of interest, $t_{m} \in\left[t_{0}, t_{0}+T\right], t_{m+1}-t_{m}=\Delta t$, $m=0,1, \ldots, M$.

3) The mean solution $\overline{\boldsymbol{x}}\left[t_{m} \mid \overline{\boldsymbol{x}}\left(\tau_{i-1}\right)\right]$ at time $\tau_{i-1} \leq t_{m}<\tau_{i}$ is obtained by propagating forward the TLE $\overline{\boldsymbol{x}}\left(\tau_{i-1}\right)$ immediately preceding $t_{m}$ with SGP4 until reaching $t_{m}$. This is done for all $t_{m} \in\left[\tau_{i-1} \leq t_{m}<\tau_{i}\right]$.

4) The osculating solution at time $t_{m}$ is obtained by adding the short-periodic terms to the SGP4 mean solution at time $t_{m}$,

$$
\boldsymbol{x}\left(t_{m}\right)=\overline{\boldsymbol{x}}\left(t_{m}\right)+\delta \boldsymbol{x}\left[\overline{\boldsymbol{x}}\left(t_{m}\right)\right] .
$$

In the above equation, $\delta \boldsymbol{x}$ are the short-periodic terms detailed in appendix B.B.7 of Ref. [8].

5) Steps 3 and 4 are repeated for all $t_{m} \in\left[t_{0}, t_{0}+T\right]$.

The synthesised solution is not continuous, as small jump discontinuities are introduced after each TLE.

The generation of a synthetic solution valid for arbitrary time spans and intervals allows us to investigate its consistency during the last two decades. For this purpose, we compare the synthetic solution obtained built with the procedure outlined above to an osculating solution obtained from the Horizons system 8 At each epoch, the Horizons

\footnotetext{
${ }^{\ddagger}$ Here and in the rest of the paper, we consider frequencies to be expressed in cycles per interval, which is equivalent to $2 \pi$ radians per interval. ${ }^{\S}$ URL: https://ssd.jpl.nasa.gov/horizons/ Last visited: $30^{\text {th }}$ November 2021.
} 

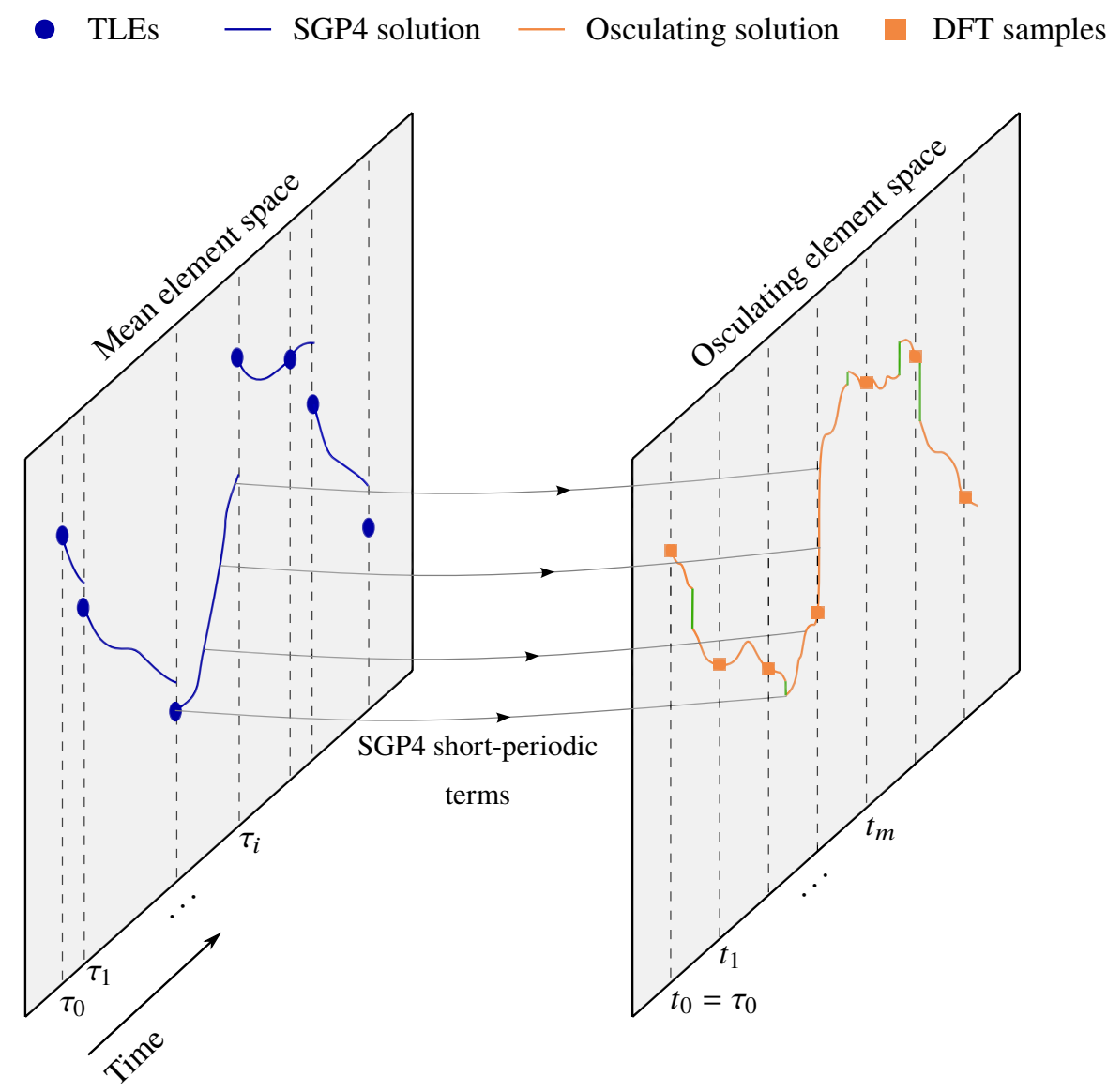

Fig. 3 Generation of a piecewise osculating solution from a mean SGP4 solution. Jump discontinuities in the osculating solution are shown in green.

solution is obtained a weighted interpolation of backward and forward SGP4 propagations from adjacent TLEs 1 and is thus conceptually similar to the synthetic solution. Both solutions cover a 25-year time span, from $24^{\text {th }}$ February 1996 until $24^{\text {th }}$ February 2021, and the synthetic solution is rotated from the TEME frame to the ICRF frame for comparison [10].

Three-month moving averages of the radial, in-track, and cross-track components of the position and velocity differences between the two solutions are shown in fig. 4 , where we excluded a few outliers with very large differences. Note that the error magnitudes do not reflect the accuracy of the TLEs over this time span and can only serve as a proxy, as both solutions are ultimately derived from TLEs. However, as the synthetic and Horizons solutions are obtained following different procedures, the error trends hint towards the intrinsic consistency of the TLEs, which is affected by their accuracy. In particular, a change in the error trend is observed between March 2013 and March 2015, followed by a sudden reduction by a factor of 5 after March 2015. This may be due to the implementation of the numerical extrapolation of TLEs from special perturbations, and is in accordance both with published timelines for the system [19] and with other recent studies on TLE accuracy; in particular, Racelis and Joerger [33] note similar changes in error behaviour for GPS and WAAS satellites in October 2015.

\section{LAGEOS-1 trajectory in time and frequency domains}

In this section, we first perform a qualitative study of the dynamics of LAGEOS-1 in the time domain. The main dynamical features of the trajectory in the time domain are used to inform the analysis in the frequency domain, which relies on the estimation of the amplitude spectra of the time series of orbital elements.

\footnotetext{
IIJon D. Giorgini (JPL), personal communication, 2021.
} 

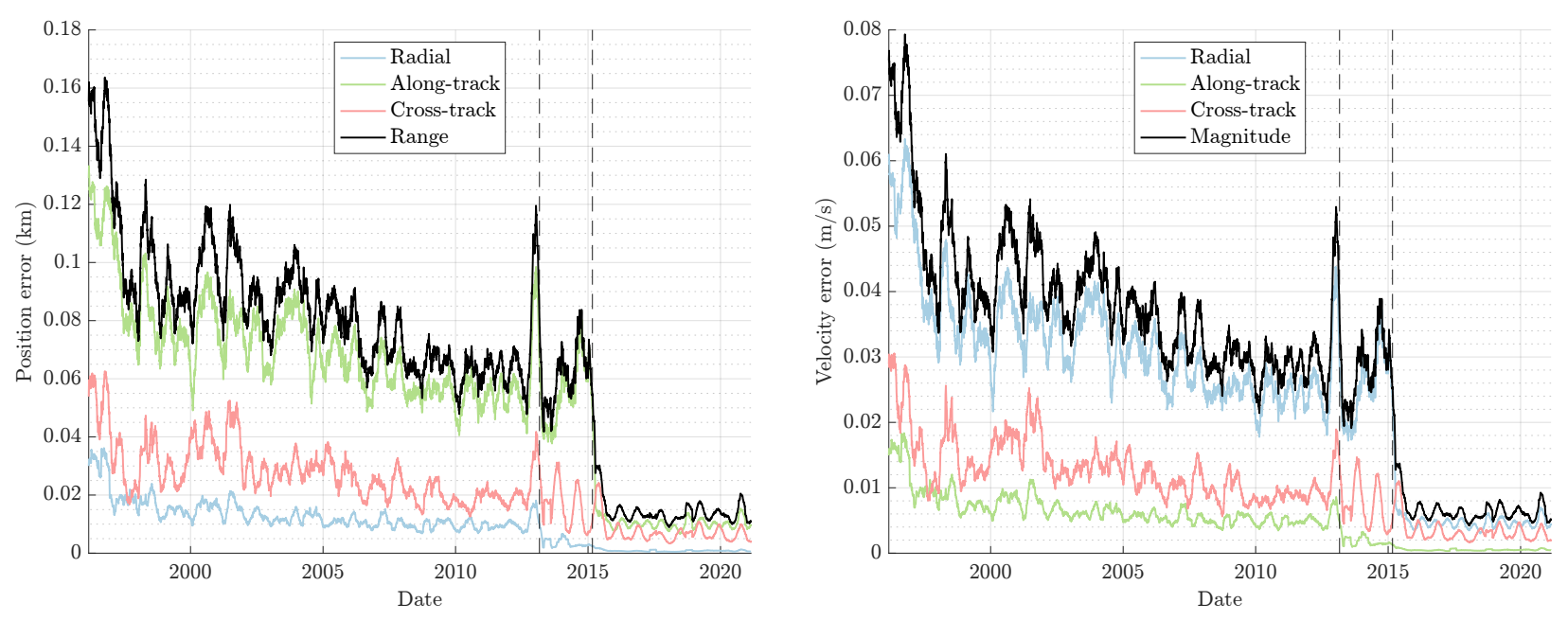

Fig. 4 Three-month moving averages of position and velocity errors of LAGEOS-1 piecewise osculating solution with respect to a solution from the Horizons system.

\section{A. Time domain}

The osculating orbital elements for LAGEOS-1 from February $24^{\text {th }} 1996$ to February $24^{\text {th }} 2021$ are shown in fig. 5. We omit showing the behaviour of the argument of perigee and of the fast variable as they would not give any useful information over the time span of interest. The solution is retrieved from the Horizons system, The osculating semi-major axis oscillates around the mean value $\bar{a}=12270 \mathrm{~km}$ due to oblateness perturbations with an amplitude of about $5 \mathrm{~km}$. The mean semi-major axis is constant, as the satellite is subject to no dissipative forces and is well inside the Earth's sphere of influence. The eccentricity and inclination show both short- and long-periodic trends. The main long-period harmonic of the eccentricity has a period of about 4.6 years and is consistent with the rotation rate of the free eccentricity [34, Ch. 7] as given by Cook [35]. Both the right ascension of ascending node and the argument of perigee have linear secular behaviours that are due to first order perturbations from $J_{2}$.

\section{B. Amplitude spectra}

To assess the presence of short-periodic terms in the time series of orbital elements for LAGEOS-1, we build an amplitude spectrum through the discrete Fourier transform (DFT).

To improve the quality of the amplitude spectrum and remove a peak at zero frequency, the transformed time series must have zero mean and no secular behaviour. We first remove the mean from the semi-major axis, eccentricity, and inclination,

$$
x_{a}=a(t)-\bar{a}, \quad x_{e}=e(t)-\bar{e}, \quad x_{i}=i(t)-\bar{i},
$$

where $a(t), e(t), i(t)$ are defined over the interval $t \in\left[t_{0}, t_{0}+T\right]$, and $\bar{a}, \bar{e}, \bar{i}$ are the means of the respective elements over the considered time span. As both the RAAN and argument of perigee grow secularly, we remove the linear behaviour by considering

$$
\begin{aligned}
& x_{\Omega}=\Omega(t)-\left[\frac{\Omega_{\mathrm{u}}\left(t_{0}+T\right)-\Omega\left(t_{0}\right)}{T}\left(t-t_{0}\right)+\Omega\left(t_{0}\right)\right] \\
& x_{\varpi}=\varpi(t)-\left[\frac{\varpi_{\mathrm{u}}\left(t_{0}+T\right)-\varpi\left(t_{0}\right)}{T}\left(t-t_{0}\right)+\varpi\left(t_{0}\right)\right],
\end{aligned}
$$

where $\Omega_{\mathrm{u}}, \varpi_{\mathrm{u}}$ are the "unwrapped" RAAN and argument of perigee, obtained by adding $360^{\circ}$ to the respective angle value at each zero crossing.

We compute the DFT of the zero-mean time series of orbital elements,

$$
X\left(\omega_{k}\right)=\sum_{m=0}^{M-1} x\left(t_{m}\right) e^{-j 2 \pi m \Delta t \omega_{k}},
$$



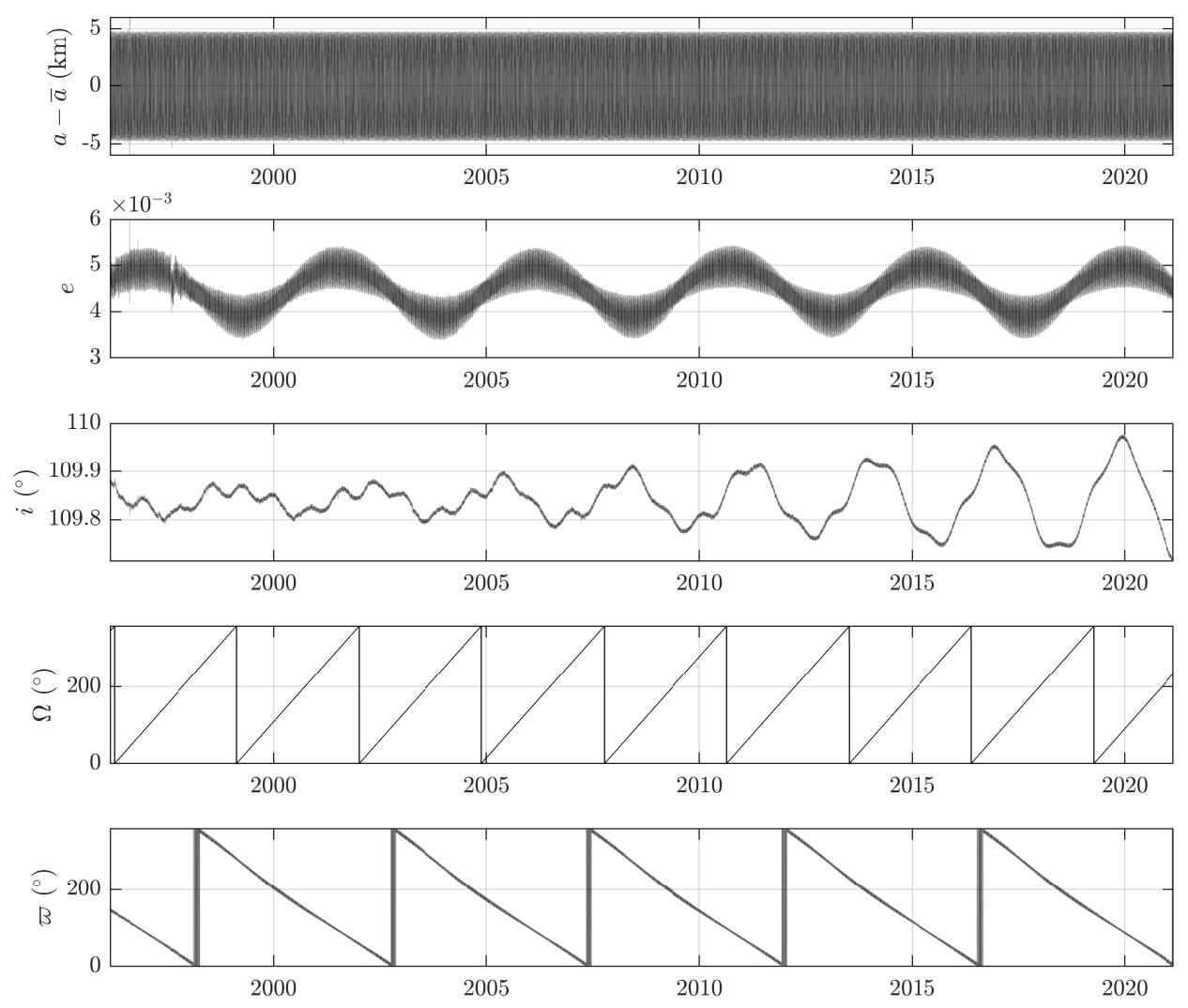

Fig. 5 Slow orbital elements derived as a function of time for LAGEOS-1 from 24-Feb-1996 to 24-Feb-2021. The mean semi-major axis is $\bar{a}=12270 \mathrm{~km}$. 
where $M$ is the number of samples, $t_{m}=t_{0}+m \Delta t, x$ is any of $x_{a}, x_{e}, x_{i}, x_{\Omega}$, and $\omega_{k}$ is the $k$ th Fourier frequency,

$$
\omega_{k}=\frac{k}{M \Delta t},
$$

with $k$ ranging from $-(M+1) / 2+1$ to $(M+1) / 2$. The DFT in eq. (3) is a complex sequence defined over the primary frequency domain $\omega_{k} \in\left[-\omega_{\mathrm{c}}+1 /(2 M \Delta t), \omega_{\mathrm{c}}\right]$ and is periodic with period $1 / \Delta t$. It is straightforward to show that, because the sequence $x\left(t_{n}\right)$ is real,

$$
X^{*}\left(\omega_{k}\right)=X\left(-\omega_{k}\right),
$$

where $X^{*}$ denotes the complex conjugate [30, Ch. 3]. For the two-sided DFT introduced in eq. (3) the amplitude spectrum $A(\omega)$ of the time series will be equally distributed over negative and positive frequencies. However, as we are interested exclusively in positive frequencies, we consider the one-sided amplitude spectrum

$$
A\left(\omega_{k}\right)=\frac{1}{N}\left|X\left(-\omega_{k}\right)+X\left(\omega_{k}\right)\right|=\frac{2}{N}\left|X\left(\omega_{k}\right)\right|, \quad \omega_{k}>0
$$

in virtue of eq. (5). The amplitude spectrum is a useful tool for the discovery of periodic behaviour in time series, as harmonic components at frequency $\omega$ in a time series will appear as Dirac deltas in the amplitude spectrum that are centred on their respective frequencies.

We analyse time series of the orbital elements for the time interval $\left[t_{0}, t_{0}+T\right]$, where $t_{0}=24 \mathrm{Jul} \mathrm{2015} \mathrm{05:28:18}$ UTC and $T=5.0102$ years. We use the $\mathrm{fft}$ function in MATLAB R2021b to compute the DFTs of the zero-mean time series of orbital elements. The time series of the slow orbital elements $a(t), e(t), i(t), \Omega(t)$ are obtained by building a piecewise continuous osculating solution from TLEs through the procedure detailed in section[II for a uniform sampling interval $\Delta t=36 \mathrm{~min}$. The total number of samples is $M=73200$. As the period of LAGEOS-1 is about $225 \mathrm{~min}$, this choice of sampling interval implies a Nyquist frequency $\omega_{\mathrm{c}}=1 /(2 \Delta t) \approx 3 n$, where $n$ is the mean motion of the satellite. The Nyquist frequency thus corresponds to the highest frequency of the short-periodic terms given in Brouwer's solution of the main problem [1].

Figure 6 displays the amplitude spectra of the time series orbital elements; we show frequencies as multiples of the mean motion $n, 2 n$ to aid the interpretation. All the orbital elements have short-periodic harmonics at frequencies $n, 2 n, 3 n$, which are consistent with Brouwer's solution. However, SGP4 only retains short-periodic terms in Brouwer's solution that do not include the eccentricity as a factor [8], whose maximum frequency for a satellite on a quasi-circular orbit is $2 n$. Because of the presence of harmonics at $\omega>2 n$ in all orbital elements, we deduce that the piecewise osculating solution obtained from TLEs contains additional short-periodic terms that are inconsistent with the SGP4 theory. We speculate two origins for such periodic terms. The first is the presence of jumps in the piecewise continuous solution due to the introduction of new TLEs, which correspond to the introduction of new information in the time series. The second is due to the TLEs themselves: as the TLEs are nowadays obtained as a numerical fit to a special perturbations solution, it seems reasonable that such fit introduces effectively short-periodic terms. Effectively, the numerical fit introduces short-periodic terms in the TLEs.

Further analysis of figs. 5and 6, shows that the eccentricity and inclination, along with the non-secular part of the right ascension of ascending node, are dominated by long-periodic terms. These are hard to glean in fig. 6 , as we selected the frequency range and resolution to focus on high frequencies. Nevertheless, larger amplitude densities close to $\omega \approx 0$ are clearly visible in fig. 6 .

The amplitude spectral estimator defined through eq. (6) is sufficiently accurate for the present analysis, as the amplitudes are consistent with the behaviour of the orbital elements in the time domain in fig. 5. However, the high dynamic range in the frequency domain leads to leakage of amplitudes into adjacent frequencies, which degrades the accuracy of the amplitude spectrum. More accurate spectral estimators can be obtained by adopting appropriate windows, as explained in Ref. [30].

\section{Conclusions}

During the last decade, TLEs have been obtained by fitting the corresponding osculating elements (derived after applying SGP4 short-periodic terms) to SP solutions. We conjecture that this process effectively results in the embedding of short-periodic terms into TLEs, which should thus be considered as a type of osculating elements. To verify this conjecture, we analyse the amplitude spectrum of time series of osculating orbital elements derived from TLEs for the LAGEOS-1 satellite. However, the time interval between TLE updates is generally larger than the orbital period and 


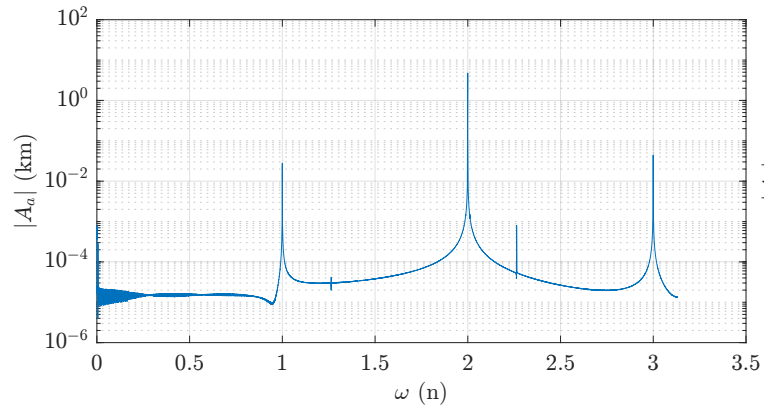

(a) Semi-major axis

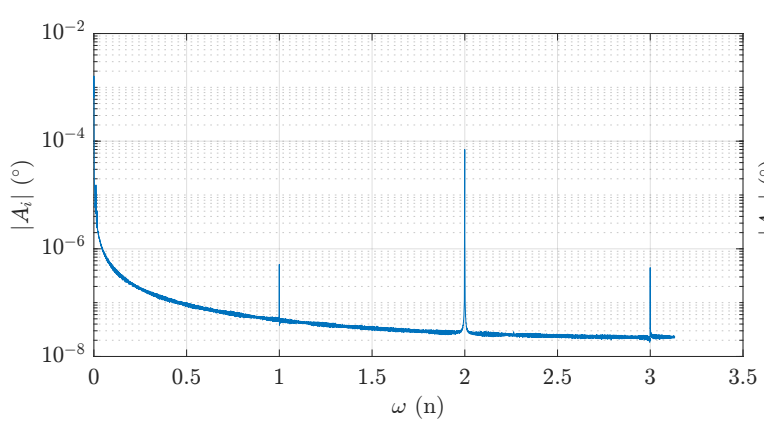

(c) Inclination

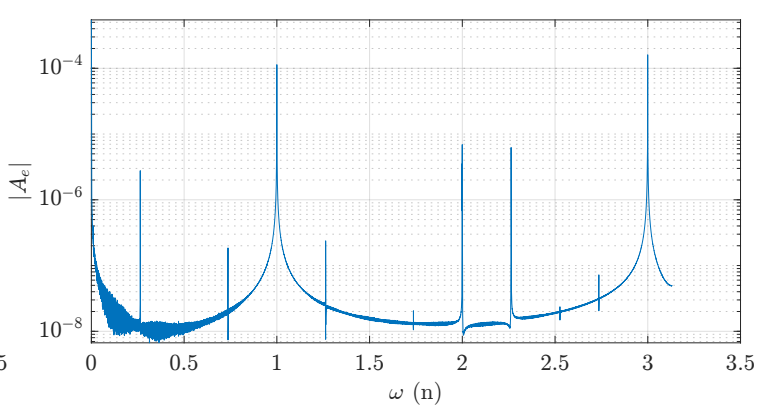

(b) Eccentricity

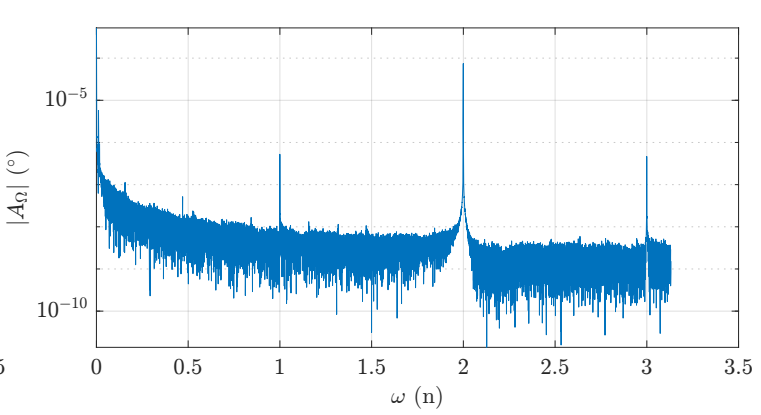

(d) RAAN

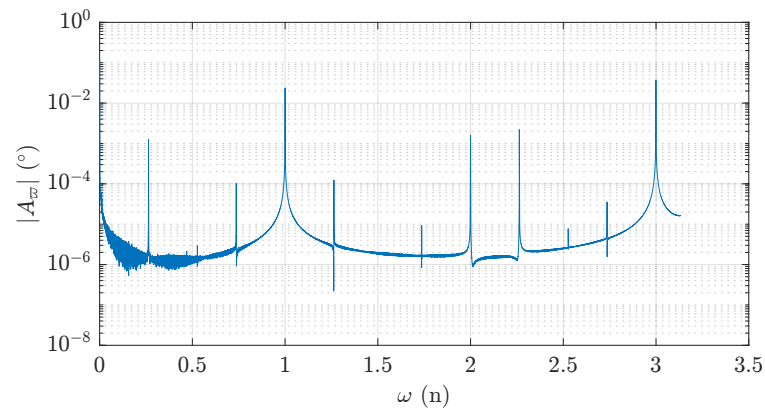

(e) Argument of perigee

Fig. 6 Amplitude spectra of the slow orbital elements for the piecewise osculating solution obtained from TLEs for LAGEOS-1. Frequencies are expressed as multiples of the mean motion. 
not uniform, which prevents the straightforward application of spectral analysis methods based on the DFT to TLE sequences. Therefore, we perform the spectral analysis on a dense piecewise continuous osculating solution generated with a uniform timestep by propagating TLEs with SGP4. The amplitude spectrum of the slow orbital elements contains high-frequency terms at frequencies larger than $3 n$, which are in addition to those reconstructed analytically by SGP4. The additional high-frequency components may also be engendered by the jump discontinuities arising at TLE epochs in the piecewise osculating solution. These results are particularly relevant for pseudo-orbit determination schemes for the improvement of TLE accuracy, which are also based on piecewise osculating solutions. In fact, it would be prudent to avoid trying to improve the accuracy of such solutions by adding short-periodic terms not reconstructed by SGP4, as this would result in "double-counting".

We propose extending the spectral analysis in time by employing time-frequency analysis methods such as spectrograms, which would be particularly helpful to understand the frequency content of orbits affected by significant dissipative and third-body perturbations. Moreover, the study should be extended to several objects across different orbital regimes in order to draw more general conclusions.

\section{Acknowledgements}

D. A. Amato is grateful to David Vallado for addressing him towards important references on numerical extrapolation of TLEs to fit special perturbations vector, Jon D. Giorgini for his kind explanation of the interpolation process of Horizons spacecraft ephemerides, and Fabien Gachet and Aaron J. Rosengren for bringing his attention to SGP4-XP.

\section{References}

[1] Brouwer, D., "Solution of the problem of artificial satellite theory without drag," The Astronomical Journal, Vol. 64, 1959, pp. 378-396. https://doi.org/10.1086/107958

[2] Kozai, Y., "The motion of a close earth satellite,” The Astronomical Journal, Vol. 64, 1959, p. 367.https://doi.org/10.1086/107957, URL http://adsabs.harvard.edu/cgi-bin/bib_query?1959AJ....64..367K.

[3] Brouwer, D., and Hori, G.-i., "Theoretical Evaluation of Atmospheric Drag Effects in the Motion of an Artificial Satellite," The Astronomical Journal, Vol. 66, No. 5, 1961, pp. 193-225. URL http://articles.adsabs.harvard.edu/pdf/1961AJ....66..193B

[4] Lyddane, R. H., "Small eccentricities or inclinations in the Brouwer theory of the artificial satellite," The Astronomical Journal, Vol. 68, 1963, p. 555. https://doi.org/10.1086/109179. URL http://adsabs.harvard.edu/cgi-bin/bib_query?1963AJ....68..555L

[5] Lane, M., "The development of an artificial satellite theory using a power-law atmospheric density representation," $2 n d$ Aerospace Sciences Meeting, American Institute of Aeronautics and Astronautics, New York,NY,U.S.A., 1965. https: //doi.org/10.2514/6.1965-35 URL https://arc.aiaa.org/doi/10.2514/6.1965-35, aIAA 65-35.

[6] Lane, M., and Cranford, K., "An improved analytical drag theory for the artificial satellite problem," American Institute of Aeronautics and Astronautics, 1969. https://doi.org/10.2514/6.1969-925, URL https://arc.aiaa.org/doi/abs/10.2514/6.1969-925

[7] Lane, M. H., and Hoots, F. R., "Spacetrack Report No. 2: General Perturbations Theories Derived from the 1965 Lane Drag Theory," Special Astrodynamic Report 2, United States Air Force, Peterson AFB, CO, USA, Dec. 1979.

[8] Hoots, F. R., Schumacher, P. W., and Glover, R. A., "History of Analytical Orbit Modeling in the U. S. Space Surveillance System," Journal of Guidance, Control, and Dynamics, Vol. 27, No. 2, 2004, pp. 174-185. https://doi.org/10.2514/1.9161. URL https://arc.aiaa.org/doi/10.2514/1.9161.

[9] Hoots, F. R., and Roehrich, R. L., “Spacetrack Report No. 3,” Tech. rep., Dec. 1980.

[10] Vallado, D., Crawford, P., Hujsak, R., and Kelso, T. S., "Revisiting Spacetrack Report \#3," American Institute of Aeronautics and Astronautics, 2006. https://doi.org/10.2514/6.2006-6753. URL https://arc.aiaa.org/doi/abs/10.2514/6.2006-6753

[11] Group, A. S. E., “Astrodynamics Standards Version 8.0 Release Notes,”, Oct. 2020.

[12] Izsak, I., "A Note on Perturbation Theory," The Astronomical Journal, Vol. 68, No. 8, 1963, pp. 559-561. URL https: //adsabs.harvard.edu/pdf/1963AJ.....68..559I

[13] Frazer, J., "On the motion of an artificial Earth satellite," Tech. Rep. ESD-TR-66-293, The Mitre Corporation, L.G. Hanscom Field, Bedford, MA, USA, Sep. 1966. 
[14] Hoots, F. R., Schumacher, P. W., and DeVere, T., "50 years of satellite tracking and cataloging in the US," Advances in the Astronautical Sciences, Advances in the Astronautical Sciences, Vol. 129, Univelt, 2008. Medium: Verschiedene Materialien Meeting Name: AAS/AIAA Astrodynamics Specialist Conference.

[15] Coffey, S., Healy, L., and Neal, H., "Applications of parallel processing to astrodynamics," Celestial Mechanics and Dynamical Astronomy, Vol. 66, No. 1, 1996, pp. 61-70. https://doi.org/10.1007/BF00048824 URL https://doi.org/10.1007/BF00048824.

[16] 18th Space Control Squadron, “Spaceflight Safety Handbook for Satellite Operators,”, Aug. 2020.

[17] Herriges, D. L., "NORAD General Perturbation Theories: an Independent Analysis," Master's thesis, Massachussetts Institute of Technology, Cambridge, Massachusetts, USA, Jan. 1988.

[18] Cappellucci, D. A., "Special perturbations to general perturbations extrapolation differential correction in satellite catalog maintenance," Astrodynamics 2005, Advances in the Astronautical Sciences, Vol. 123, Univelt, Inc., South Lake Tahoe, CA, USA, 2006.

[19] Hejduk, M. D., Casali, S. J., Cappellucci, D. A., Ericson, N. L., and Snow, D. E., "A catalogue-wide implementation of general perturbations orbit determination extrapolated from higher order orbital theory solutions," Advances in the Astronautical Sciences, Vol. 148, Univelt, Inc., Kauai, Hawaii, USA, 2013. AAS 13-240.

[20] Vallado, D., and Crawford, P., "SGP4 Orbit Determination," American Institute of Aeronautics and Astronautics, 2008. https://doi.org/10.2514/6.2008-6770 URL https://arc.aiaa.org/doi/abs/10.2514/6.2008-6770

[21] Laskar, J., "Secular evolution of the solar system over 10 million years," Astronomy \& Astrophysics, Vol. 198, 1988, pp. 341-362. URL http://adsabs.harvard.edu/pdf/1988A\&A...198..341L

[22] Laskar, J., "A numerical experiment on the chaotic behaviour of the Solar System," Nature, Vol. 338, No. 6212, 1989, pp. 237-238. https://doi.org/10.1038/338237a0. URL https://www.nature.com/articles/338237a0, bandiera_abtest: a Cg_type: Nature Research Journals Number: 6212 Primary_atype: Research Publisher: Nature Publishing Group.

[23] Knežević, Z., and Milani, A., "Synthetic Proper Elements for Outer Main Belt Asteroids," New Developments in the Dynamics of Planetary Systems, edited by R. Dvorak and J. Henrard, Springer Netherlands, Dordrecht, 2001 , pp. 17-46. https://doi.org/10.1007/978-94-017-2414-2_2

[24] Knežević, Z., and Milani, A., "Proper element catalogs and asteroid families," Astronomy \& Astrophysics, Vol. 403, No. 3, 2003, pp. 1165-1173. https://doi.org/10.1051/0004-6361:20030475. URL https://www.aanda.org/articles/aa/abs/2003/21/ aa3507/aa3507.html, number: 3 Publisher: EDP Sciences.

[25] Ely, T. A., “Transforming Mean and Osculating Elements Using Numerical Methods,” The Journal of the Astronautical Sciences, Vol. 62, No. 1, 2015, pp. 21-43. https://doi.org/10.1007/s40295-015-0036-2. URL http://link.springer.com/10.1007/s40295015-0036-2

[26] Dell'Elce, L., Caillau, J.-B., and Pomet, J.-B., “Averaging Optimal Control Systems with Two Fast Variables,” Tech. Rep. hal-01793704, Inria, May 2018. URL https://hal.inria.fr/hal-01793704

[27] Rosengren, A. J., Amato, D., Bombardelli, C., and Jah, M. K., "Resident space object proper orbital elements," Maui, Hawaii, USA, 2018.

[28] Wu, D., and Rosengren, A. J., “Analysis of Proper Orbital Element for Resident Space Objects,” Washington, D.C., USA, 2019. Paper IAC-19.C1.3.11.

[29] Celletti, A., Pucacco, G., and Vartolomei, T., "Reconnecting groups of space debris to their parent body through proper elements," Scientific Reports, Vol. 11, No. 1, 2021, p. 22676. https://doi.org/10.1038/s41598-021-02010-x. URL https: //www.nature.com/articles/s41598-021-02010-x bandiera_abtest: a Cc_license_type: cc_by Cg_type: Nature Research Journals Number: 1 Primary_atype: Research Publisher: Nature Publishing Group Subject_term: Applied mathematics;Scientific data Subject_term_id: applied-mathematics;scientific-data.

[30] Percival, D. B., and Walden, A. T., Spectral analysis for physical applications: multitaper and conventional univariate techniques, Cambridge University Press, Cambridge ; New York, NY, USA, 1993.

[31] Levit, C., and Marshall, W., "Improved orbit predictions using two-line elements," Advances in Space Research, Vol. 47, No. 7, 2011, pp. 1107-1115. https://doi.org/10.1016/j.asr.2010.10.017. URL http://www.sciencedirect.com/science/article/pii/ S0273117710006964 
[32] Vallado, D. A., and Cefola, P. J., “Two-Line Element Sets - Practice and Use,” Naples, Italy, 2012.

[33] Racelis, D., and Joerger, M., "High-Integrity TLE Error Models for MEO and GEO Satellites," 2018 AIAA SPACE and Astronautics Forum and Exposition, American Institute of Aeronautics and Astronautics, Orlando, FL, 2018. https: //doi.org/10.2514/6.2018-5241. URL https://arc.aiaa.org/doi/10.2514/6.2018-5241

[34] Murray, C. D., and Dermott, S. F., Solar System Dynamics, Cambridge University Press, Cambridge, 2000. https://doi.org/10. 1017/CBO9781139174817. URL http://ebooks.cambridge.org/ref/id/CBO9781139174817.

[35] Cook, G. E., "Perturbations of near-circular orbits by the Earth's gravitational potential," Planetary and Space Science, Vol. 14, 1966, pp. 433-444. 